\title{
Factors Influencing the Success Rate of Cardiopulmonary Resuscitation
}

\author{
Aisyah Amanda Hanif, ${ }^{1}$ Iwan Abdul Rachman, ${ }^{2}$ Hendro Sudjono Yuwono ${ }^{3}$ \\ ${ }^{1}$ Faculty of Medicine Universitas Padjadjaran, ${ }^{2}$ Department of Anesthesiology and Intensive \\ Care Faculty of Medicine Universitas Padjadjaran/Dr. Hasan Sadikin General Hospital Bandung, \\ ${ }^{3}$ Department of Surgery Faculty of Medicine Universitas Padjadjaran/Dr. Hasan Sadikin General \\ Hospital Bandung
}

\begin{abstract}
Background: Cardiopulmonary resuscitation (CPR) is a series of actions performed on cardiac arrest patients. Not all patients receiving CPR can survive. The outcome of CPR is influenced by several factors. This study was conducted to determine the success rate of CPR and the factors influencing it in Dr. Hasan Sadikin General Hospital in 2013.

Methods: This study was conducted by using 168 patient medical records who underwent CPR and met the inclusion criteria in the Resuscitation Room of Dr. Hasan Sadikin General Hospital from January to December 2013. The collected data consisted of age, gender, pre-arrest diagnosis, initial rhythm, response time and clinical outcome of CPR. The results were expressed in frequencies and percentage. The data were analyzed using the chi-square test.

Results: The Success rate of CPR was $15.5 \%$. The success rate was higher in patients with cardiac prearrest diagnoses $(8.33 \%, \mathrm{p}=0.024)$. The most common initial rhythm was unshockable rhythms $(83.92 \%)$, yet patients with shockable heart rhythms had higher success rates $(40.74 \%, \mathrm{p}<0.001)$. All of the surviving patients had response time within the first minute from cardiac arrest.

Conclusions: Success rate of CPR in the resuscitation room of Dr. Hasan Sadikin General Hospital during 2013 is still low. The factors influencing the survival rate are the pre-arrest diagnosis and initial heart rhythm. [AM].2015;2(4):615-9]
\end{abstract}

Keywords: Cardiopulmonary resuscitation, influencing factors, success rate

\section{Introduction}

Cardiac arrest is a condition in which there is an abrupt cessation of cardiac mechanical function. This condition may be reversible with a prompt intervention, and may cause death if the intervention is not delivered. ${ }^{1}$ Cardiac arrest is managed using basic life supports which consists of activation of emergency response system, cardiopulmonary resuscitation (CPR) and defibrillation with an automated external defibrillator (AED). ${ }^{2}$

In a hospital setting, advanced cardiac life support is used. The goal of CPR is to maintain the viability of vital organs until a definitive intervention can be done. To achieve this goal, resuscitation is done in a series of steps, those are, chest compressions, airway maintenance, and giving rescue breaths or ventilations. ${ }^{2}$ However, chest compression only produces below $30 \%$ of the normal blood flow. ${ }^{3}$ This is one of the reasons why CPR does not always yield a positive outcome.

Studies in Turkey ${ }^{4}$ and $\operatorname{Iran}^{5}$ reported that $13.4 \%$ and $12 \%$ patients survived until discharge. Another study conducted in Turkey and Iran had an immediate survival of $49.3 \%$ and $19.9 \%{ }^{5,6}$ Several factors are contributed to the outcome of CPR. Literatures stated that the mechanism of cardiac arrest, initial rhythm after cardiac arrest, gender, clinical setting, response time, time of day during cardiac arrest, and duration of CPR are some of the few factors that influence the outcome of CPR. ${ }^{1,45,7}$ The aim of this study was to determine the factors influencing the success rate of cardiopulmonary resuscitation in Dr. Hasan Sadikin General Hospital during 2013.

\section{Methods}

This analytical observational study was

Correspondence: Aisyah Amanda Hanif, Faculty of Medicine, Universitas Padjadjaran, Jalan Raya Bandung-Sumedang Km.21, Jatinangor, Sumedang, Indonesia, Phone: +62 81312371875 Email: aisyahahs@gmail.com 
conducted by obtaining data from resuscitation reports and medical records of patients receiving CPR in the resuscitation room of $\mathrm{Dr}$. Hasan Sadikin General Hospital from January to December 2013. This study was approved by the Ethical Clearance Committee and data collection started from August to October 2014.

The inclusion criteria were patients receiving CPR in the resuscitation room of Dr. Hasan Sadikin General Hospital in 2013, listed in the resuscitation report, and has a medical record containing the variables of interest. Patients with do not resuscitate (DNR) orders, refusal of family to continue CPR, patients who arrived at the resuscitation room in a death on arrival (DOA) condition and patients with medical records lacking the data needed were excluded. A total of 168 medical records were enrolled in this study.

The clinical outcome at the end of CPR, prearrest diagnosis, initial rhythm, and response time were collected from resuscitation reports and medical records. Clinical outcome at the end of CPR was categorized into successful and unsuccessful. Successful CPR was defined as the patient leaving the resuscitation room was still alive. Pre-arrest diagnoses were categorized into cardiac and non-cardiac, with non-cardiac diagnoses including respiratory disease, renal disturbance, sepsis, trauma, and cancer. Initial heart rhythms were defined as shockable rhythms (ventricular fibrillation (VF) or pulseless ventricular tachycardia (PVT)) and unshockable heart rhythms (asystole or pulsesless electrical activity (PEA)). Response time was defined as the period of time from cardiac arrest until the start of CPR. Response time was categorized into below 1 minute and above 1 minute.

These data were then processed using a statistics computer program. The obtained results were expressed in frequencies and percentage. The significance of influencing factors were evaluated using the Chi-square test. A particular influencing factor is considered significantly if the $p$-value was less than 0.05 .
Table 1 General Characteristics of Patients Receiving CPR

\begin{tabular}{lc}
\hline \multicolumn{1}{c}{ Characteristic } & Frequency $\mathbf{n}(\%)$ \\
\hline Age (years) & \\
$0-10$ & $12(7.1)$ \\
$11-20$ & $13(7.7)$ \\
$21-30$ & $17(10.1)$ \\
$31-40$ & $19(11.3)$ \\
$41-50$ & $29(17.3)$ \\
$51-60$ & $40(23.8)$ \\
$61-70$ & $23(13.7)$ \\
$71-84$ & $15(8.9)$ \\
Gender & \\
Male & $94(56)$ \\
Female & $74(44)$ \\
\hline
\end{tabular}

\section{Results}

The collected data from the medical records showed that the patients underwent the cardiopulmonary resuscitation were 4 days to 84 years old (most were above 40 years old). The majority of the patients (56\%) were male. Of the 168 patients who underwent CPR, 26 patients $(15.5 \%)$ survived while 142 patients $(85.5 \%)$ were died at the end of CPR. The success rate of CPR did not differ significantly among gender and between patients below and above 60 years of age $(p=0.274$ and $\mathrm{p}=0.568$ )

Pre-arrest diagnoses were categorized as cardiac and non-cardiac. Non-cardiac includes respiratory disease, renal disturbance, sepsis, trauma, and cancer. Cardiac arrests with noncardiac as the pre-arrest diagnosis were more common $(65.48 \%)$. However, the success rate was higher in patients with cardiac conditions since before the cardiac arrest, 14 of 26 patients with successful CPR. Chi-square test was used to obtain the $p$-value $(p=0024)$,

Table 2 Outcome Distribution of CPR

\begin{tabular}{lcc}
\hline \multicolumn{1}{c}{ Outcome } & Frequency (n) & Percentage (\%) \\
\hline Successful & 26 & 15.5 \\
Unsuccessful & 142 & 84.5 \\
Total & 168 & 100 \\
\hline
\end{tabular}


Table 3 Factors Related with Successful CPR

\begin{tabular}{lcccc}
\hline \multicolumn{1}{c}{ Variable } & $\begin{array}{c}\text { Total Patients } \\
\text { n (\%) }\end{array}$ & $\begin{array}{c}\text { Successful } \\
\text { n (\%) }\end{array}$ & $\begin{array}{c}\text { Unsuccessful } \\
\text { n (\%) }\end{array}$ & P- value \\
\hline Age & & & & \\
$\leq 60$ years & 130 & $19(14.6)$ & $111(85.4)$ & Ns \\
$>60$ years & 38 & $7(18.4)$ & $31(81.6)$ & \\
Sex & & & & \\
$\quad$ Male & $94(56)$ & $12(12.76)$ & $82(87.23)$ & Ns \\
Female & $74(44)$ & $14(18.92)$ & $60(81.08)$ & \\
Pre-Arrest Diagnosis & & & & \\
$\quad$ Cardiac & $58(34.52)$ & $14(24.14)$ & $44(75.86)$ & 0.024 \\
$\quad$ Non-Cardiac & $110(65.48)$ & $12(10.9)$ & $98(89.1)$ & \\
Initial Heart Rhythm & & & & \\
$\quad$ Shockable (VF/PVT) & $27(16.1)$ & $11(40.74)$ & $16(59.26)$ & $<0.001$ \\
$\quad$ Unshockable (Asystole/PEA) & $141(83.9)$ & $15(10.64)$ & $126(89.36)$ & \\
Response time & & & & \\
$\quad<1$ minute & $159(94.6)$ & $26(16.35)$ & $133(83.65)$ & Ns \\
$\geq 1$ minute & $9(5.4)$ & 0 & $9(100)$ & \\
\hline
\end{tabular}

Note: $\mathrm{VF}=$ Ventricular Fibrilation, $\mathrm{PVT}=$ Pulseless Ventricular Tachychardia, $\mathrm{PEA}=$ Pulsesless Electrical Activity, $\mathrm{ns}=$ not significant

which indicated that the differences of success rate among the pre-arrest diagnoses were statistically significant.

The most common initial rhythm was unshockable heart rhythm, asystole/PEA (83.92\%) followed by shockable rhythm, VF/ PVT (16.08\%). The success rate was higher in patients with shockable rhythms (40.74\%-11 of 27 patients) than patients with unshockable rhythms $(10.63 \%-15$ of 141 patients).The $\mathrm{p}$-value was obtained using the chi-square test and indicated that the differences of success rate among the initial heart rhythms were statistically significant $(\mathrm{p}<0.001)$.

From 159 patients who received resuscitation within the first minute after cardiac arrest, only $26(16.35 \%)$ patients had succesful outcome. Moreover, all patients who received resuscitation after one minute after cardiac arrest were died.

\section{Discussion}

This study discovered that only $15.5 \%$ patients receiving CPR in the resuscitation room of Dr. Hasan Sadikin General Hospital. had survived. Compared to the studies in Turkey and Iran (49.3\% and $19.9 \%$, respectively), this result showed that a lower percentage than those studies. ${ }^{5,8}$

In this study, age and gender did not influence the CPR success rate. A study in Turkey ${ }^{5}$ with a cut-off age over 65 years found similar results where age did not significantly influence the immediate survival and discharge rate. Previous studies reported that a decrease in CPR success rate was seen in older patients. ${ }^{8}$ However, this may be caused by the co-morbidities rather than the increasing age itself. Gender did not influence the success rate and this result is similar to other studies. ${ }^{5}$ However, a study in Pakistan found that male gender was a negative predictor of return of spontaneous circulation (ROSC). ${ }^{9}$

The majority of pre-arrest diagnoses were in the non-cardiac group (65.47\%). This is in accordance to the previous study in Iran that found only $19.2 \%$ CPR was caused by cardiac disease. ${ }^{4}$ However, in this study, 14 of the 26 successful CPR happened in the cardiac as the pre-arrest diagnosis group, showing a small difference between the cardiac and noncardiac group. This result was different from the previous study in San Juan that found most CPR survivors suffered cardiac arrest of primarily respiratory origin..$^{10}$ Another study reviewing literatures about in-hospital cardiac arrest (IHCA) found that some pre-arrest conditions such as cancer, sepsis and renal 
failure were correlated with lower survival. ${ }^{8}$ A meta-analysis about predictors of failure to survive after CPR found that cardiovascular diagnoses and co-morbidities (arrhythmia, admission for cardiovascular disease and hypertension) were associated with a higher chance of survival. ${ }^{11}$

The most common initial rhythm was unshockable heart rhythms, asystole/PEA $(83.92 \%)$ followed by shockable rhythms, VF/PVT (16.08\%). The success rate was higher in patients with shockable rhythms (40.74\%-11 of 27 patients) than in patients with unshockable rhythms $(10.63 \%-15$ of 141 patients). Previous studies had similar results where the majority of cases had an initial rhythm of asystole/PEA but the success rate itself was higher in VF/PVT. ${ }^{8-10,12}$

Response time was categorized into below and above 1 minute. All 26 patients with successful outcomes received CPR within the first minute after cardiac arrest. All of patients received CPR after 1 minute died. However, the $\mathrm{p}$-value was $>0.05$, showing that the response time did not significantly influence the success rate in this study. Study conducted in Thailand and other countries found that survival to discharge after cardiac arrest was significantly higher when CPR was given within the first minute after collapse. ${ }^{8,13}$

Limitation of this study is that many medical records lacked the data needed causing a great number of cases being excluded. A better reporting system is required so that further studies can be done to determine other factors influencing the success rate in Dr. Hasan Sadikin General Hospital and discover a way to increase the number of patients surviving CPR.

The conclusion of this study is the success rate in resuscitation room of Dr. Hasan Sadikin General Hospital during 2013 is still low $(15.5 \%)$. The factors influencing the success rate of CPR in the resuscitation room of Dr. Hasan Sadikin General Hospital are the prearrest diagnosis and the initial heart rhythm.

\section{References}

1. Myerburg RJ, Castellanos A. Cardiac arrest and sudden cardiac death. Braunwald's heart disease, a textbook of cardiovascular medicine. 9th ed. Philadelphia: Saunders; 2012. p. 845-72.

2. Berg RA, Hemphill R, Abella BS, Aufderheide TP, Cave DM, Hazinski MF, et al. Part 5: Adult basic life support: 2010 American heart association guidelines for cardiopulmonary resuscitation and emergency cardiovascular care. Circulation. 2010;122(18 suppl 3):S685S705.

3. Kern KB. Pathophysiology of Cardiac Arrest. The textbook of emergency cardiovascular care and CPR. Philadelphia: Lippincott Williams \& Wilkins; 2009. p. 149-156.

4. Saghafinia M, Motamedi MHK, Piryaie M, Rafati H, Saghafi A, Jalali A, et al. Survival after in-hospital cardiopulmonary resuscitation in a major referral center. Saudi J Anaesth. 2010;4(2):68-71.

5. Pembeci K, Yildirim A, Turan E, Buget M, Camci E, Senturk M, et al. Assessment of the success of cardiopulmonary resuscitation attempts performed in a Turkish university hospital. Resuscitation. 2006;68(2):221-9.

6. Hajbaghery MA, Mousavi G, Akbari H. Factors influencing survival after inhospital cardiopulmonary resuscitation. Resuscitation. 2005;66(3):317-21.

7. Travers AH, Rea TD, Bobrow BJ, Edelson DP, Berg RA, Sayre MR, et al. Part 4: CPR overview: 2010 American heart association guidelines for cardiopulmonary resuscitation and emergency cardiovascular care. Circulation. 2010;122(18 Suppl 3):S67684.

8. Sandroni C, Nolan J, Cavallaro F, Antonelli M. In-hospital cardiac arrest: incidence, prognosis and possible measures to improve survival. Intensive Care Med. 2007;33(2):237-45.

9. Rehmani R, Baqir M, Amanullah S. Return of spontaneous circulation and survival at hospital discharge in patients with outof-hospital and emergency department cardiac arrests in a tertiary care centre. J Pak Med Assoc. 2007:278-81.

10. Santos-Llanos G, Latalladi-Ortega G, Galera-Santiago Ã, Torres-Palacios A, Rodriguez-Cintron W. Predictors of survival in resuscitation. Crit Care \& Shock. 2008;11:54-60.

11. Ebell MH, Afonso AM. Pre-arrest predictors of failure to survive after in-hospital cardiopulmonary resuscitation: a metaanalysis. Fam Pract. 2011:505-15.

12. Kaleja A, Mikijanska D, Vanags I. Results of cardiopulmonary resuscitation during in-hospital cardiac arrest. Acta Chir Latv. 2011;11(1):84-8.

13. Siriphuwanun V, Punjasawadwong Y, Lapisatepun W, Charuluxananan 
S, Uerpairojkit K, Patumanond J. The initial success rate of cardiopulmonary resuscitation and its associated factors in patients with cardiac arrest within 24 hours after anesthesia for an emergency surgery. Risk Manag Healthc Policy. 2014; 7:65-71. 